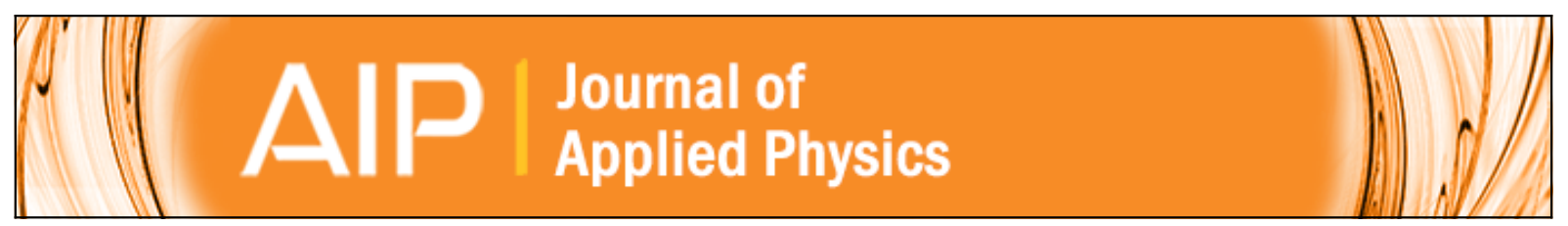

\title{
High non-linear magnetoelectric coefficient in Metglas/PMN-PT laminate composites under zero direct current magnetic bias
}

Ying Shen, Junqi Gao, Yaojin Wang, Jiefang Li, and D. Viehland

Citation: Journal of Applied Physics 115, 094102 (2014); doi: 10.1063/1.4867516

View online: http://dx.doi.org/10.1063/1.4867516

View Table of Contents: http://scitation.aip.org/content/aip/journal/jap/115/9?ver=pdfcov

Published by the AIP Publishing

\section{Articles you may be interested in}

Influence of metglas layer on nonlinear magnetoelectric effect for magnetic field detection by frequency modulation

J. Appl. Phys. 117, 024104 (2015); 10.1063/1.4905622

Piezoelectric single crystal and magnetostrictive Metglas composites: Linear and nonlinear magnetoelectric coupling

Appl. Phys. Lett. 104, 142909 (2014); 10.1063/1.4871101

Giant magnetoelectric effect in self-biased laminates under zero magnetic field

Appl. Phys. Lett. 102, 082404 (2013); 10.1063/1.4794056

Enhancement in magnetic field sensitivity and reduction in equivalent magnetic noise by magnetoelectric laminate stacks

J. Appl. Phys. 111, 104504 (2012); 10.1063/1.4718441

Magnetoelectric coupling of laminated composites under combined thermal and magnetic loadings

J. Appl. Phys. 111, 023906 (2012); 10.1063/1.3677945

MIT LINCOLN

LABORATORY CAREERS

Discover the satisfaction of innovation and service

to the nation
- Space Control

- Air \& Missile Defense

- Communications Systems \& Cyber Security

- Intelligence, Surveillance and

Reconnaissance Systems

- Advanced
Electronics
- Tactical Systems
" Homeland
Protection
- Air Traffic Control

LINCOLN LABORATORY

MASSACHUSETTS Institute OF TeChNOLOGY

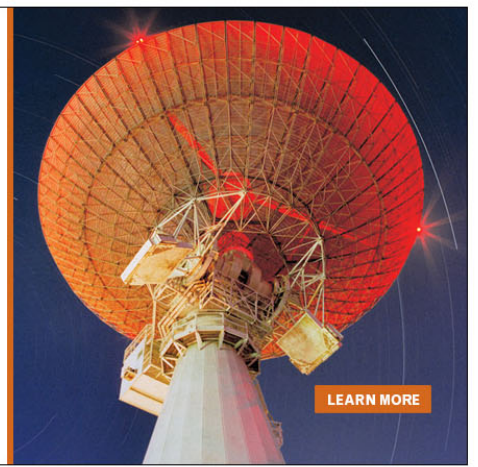




\title{
High non-linear magnetoelectric coefficient in Metglas/PMN-PT laminate composites under zero direct current magnetic bias
}

\author{
Ying Shen, Junqi Gao, Yaojin Wang, Jiefang Li, and D. Viehland \\ Department of Materials Science and Engineering, Virginia Tech, Blacksburg, Virginia 24061, USA
}

(Received 1 February 2014; accepted 5 February 2014; published online 4 March 2014)

\begin{abstract}
The non-linear magnetoelectric (ME) response of Metglas/PMN-PT based sandwiched ME laminate composites has been studied for various thicknesses of the magnetostrictive layer. A significant increase in the non-linear ME coefficient under zero direct current bias was observed with a decreased Metglas thickness ratio for a fixed number of Metglas layers of $n=2$. The non-linear ME effect was further improved by driving the laminate at the electromagnetic resonant frequency. The approach offers the potential to modulate low frequency magnetic signals to higher frequencies, where the noise floor is much lower and the signal to noise ratio higher. (C) 2014 AIP Publishing LLC. [http://dx.doi.org/10.1063/1.4867516]
\end{abstract}

\section{INTRODUCTION}

The existence of a cross-coupling between the magnetic and electric orders, termed the magnetoelectric (ME) interaction, enables the control of ferroelectric polarization by a magnetic field and, conversely, the manipulation of magnetization by an electric field. ${ }^{1,2}$ Composite ME materials comprised of alternating ferromagnetic and ferroelectric phases have been found to yield ME coefficients that are larger than that of natural multiferroics by several orders of magnitude. ${ }^{3}$ The ME effect in such bilayered ME composites is strain mediated, i.e., the strain induced in the ferromagnetic phase due to its magnetostriction is transferred to the ferroelectric phase and alters its polarization via the piezoelectric effect.

Recently, there has been interest in using the non-linear ME effect ( $\left.\alpha_{\text {nonlin }}^{\mathrm{ME}}\right)$, which has provoked a number of research activities in signal processing. For example, the non-linear ME interactions allow the realization of active mode ME sensors which can modulate weak low frequency signals to a higher frequency bandwidth. This results in the mitigation of a strong $1 / f$ noise, which is often quite large at frequencies near or below 1 Hertz $(\mathrm{Hz}){ }^{4-6}$ A DC magnetic field $\left(H_{\mathrm{DC}}\right)$ controlled frequency multiplier/doubling effect has been reported in $\mathrm{FeBSiC} / \mathrm{PZT}^{7}$ and Metglas $/ \mathrm{PZT}^{8} \mathrm{ME}$ unimorph structures. Different strengths of the geomagnetic field scalar along three mutually orthogonal directions can serve as an intelligent switch for the control of this frequency multiplication effect. ${ }^{9}$ The underlying principle of these phenomena is the same. This can be attributed to a non-linear ME effect, which is dependent on the non-linear property of the effective piezomagnetic coefficient $\left(d_{33}^{m}\right)$ that is proportional to $H^{2}$ (where $H$ is the applied magnetic field). ${ }^{10}$ In piezoelectric/magnetostrictive composites, the induced $\mathrm{ME}$ output $(V)$ is proportional to $d_{33}^{m},{ }^{11}$ resulting in $V$ being proportional to $H^{2}$. Thus, when an applied alternating current (AC) magnetic field $H_{\mathrm{AC}}$ is superimposed with a DC magnetic field bias $H_{\text {DC }}$, the total effective field in the ME composite is $H=H_{D C}+H_{A C} \sin \left(\omega_{0} t\right)$, which produces a frequency multiplication effect. By applying another incident AC field $H_{\text {inc, }}$ the applied magnetic field becomes $H=H_{D C}+H_{A C} \sin \left(\omega_{0} t\right)$
$+H_{\text {inc }} \sin \left(\omega_{1} t\right)$, and frequency modulation and mixing can be achieved.

Enhancement in the value of $\alpha_{\text {nonlin }}^{\mathrm{ME}}$ is an important consideration with regards to the practical use of the non-linear ME effect, i.e., frequency mixing and modulation. There have been limited reports for Metglas/piezo-fiber composites that exhibit $\alpha_{\text {nonlin }}^{\mathrm{ME}}$ on the order of $2.2 \mathrm{~V} /\left(\mathrm{cm}-\mathrm{Oe}^{2}\right)$ at $H_{\mathrm{DC}}=2$ Oe under a driving signal of $f_{0}=1 \mathrm{kHz}$, which can be improved to $\alpha_{\text {nonlin }}^{\mathrm{ME}}=25.3 \mathrm{~V} /\left(\mathrm{cm}-\mathrm{Oe}^{2}\right)$ by simply optimizing $f_{0}$ to be the electromechanical resonant (EMR) frequency. ${ }^{12}$ Here, an enhancement of $\alpha_{\text {nonlin }}^{\mathrm{ME}}$ at $H_{\mathrm{DC}}=0$ for a Metglas/PMN-PT heterostructure is shown through an optimized Metglas-piezofiber thickness ratio. Of particular importance, the frequency modulation scheme for the non-linear ME effect can shift low frequency signals to higher frequency ones to achieve a reduced noise floor. With regards to frequency modulation, the value of $f_{0}$ was selected to be at the EMR condition of the structure in order to optimize $\alpha_{\text {nonlin }}^{\mathrm{ME}}$ and, correspondingly, to decrease the equivalent magnetic noise floor. As a result, the signal to noise ratio (SNR) was significantly increased by a factor of 15 at $f_{0}=$ EMR relative to $f_{0}=1 \mathrm{kHz}$.

\section{EXPERIMENT AND DISCUSSION}

The PMN-PT ME laminates were comprised of two trilayers of magnetostrictive Metglas (Vacuumscheltze $\mathrm{GmbH}$ \& Co. KG, Germany) sandwiching a piezoelectric PMN-PT (Ceracomp, Korean) core. ${ }^{13}$ When an external magnetic field was applied, the Metglas layers generated a magnetic field induced strain via magnetostriction, resulting in a stress on the adjacent PMN-PT layers, which in turn produced a stress-induced electrical charge. Five PMN-PT fibers were used that had overall dimensions of $40 \times 10 \times 0.2 \mathrm{~mm}^{3}$. Both top and bottom surfaces of the core composites were fixed with a Kapton interdigitated (ID) copper electrodes layers (Smart materials, USA) using epoxy resin (Stycast 1264, USA). The copper electrodes had $500 \mu \mathrm{m}$ wide digits spaced at $1.5 \mathrm{~mm}$ center-to-center to obtain the push-pull geometry. The PMN-PT piezo-fibers were longitudinal poled. Each 
piezo-fiber had numerous alternating symmetric longitudinally poled "push-pull" units. $25 \mu \mathrm{m}$ thick Metglas foils were cut to have $10 \mathrm{~mm}$ widths, similar to the core PMN-PT layer, and $100 \mathrm{~mm}$ lengths. Previous studies have shown this geometry to have the largest value of $\alpha_{\text {nonlin }}^{\mathrm{ME}}$ due to magnetic flux concentration. ${ }^{10}$ Six Metglas layers of such dimensions were then symmetrically attached to the two sides of the core composite using another epoxy resin (West system 206, USA). To minimize the epoxy thickness in-between the layers, the laminates were then pressed by a vacuum bag and cured for more than $24 \mathrm{~h}$ at room temperature to finish fabrication of the ME laminates.

The laminates were wound with 200-turn windings, through which a $H_{\mathrm{AC}}$ signal was excited by a dynamic signal analyzer (SR-785, Stanford Research Systems, USA). This achieved an active modulation mode ME sensor, as shown in the inset of Figure 1. The active sensor unit was then placed into a Helmholtz coil driven by another signal generator with a low frequency incident signal $H_{\text {inc }}$. The induced voltages across the ID electrodes of the PMN-PT fibers at a modulation frequency of $f_{0} \pm f_{1}$ were then measured by the SR-785 dynamic signal analyzer in response to $H_{\mathrm{AC}}=0.1 \mathrm{Oe}$ at $f_{0}=1 \mathrm{kHz}$ and $H_{\text {inc }}=0.0018$ Oe at $f_{1}=1 \mathrm{~Hz}$. The Helmholtz coil together with the active sensor was placed between two poles of an electromagnet. Both DC and AC fields were directed along the longitudinal direction of the laminates, and measurements were performed as a function of DC magnetic bias with field strength of $-30 \mathrm{Oe} \leq H_{\mathrm{DC}} \leq 30 \mathrm{Oe}$.

Figure 1 shows $\alpha_{\text {nonlin }}^{\mathrm{ME}}$ as a function of $H_{\mathrm{DC}}$ for different values of $\mathrm{n}$ magnetostrictive layers stacked on both sides of the piezo-fiber core, where $\mathrm{n}$ was varied by successively peeling off layers. From this figure, it can be seen that $\alpha_{\text {nonlin }}^{\mathrm{ME}}$ was maximum at $H_{\mathrm{DC}}=0$; decreased rapidly as $H_{\mathrm{DC}}$ was increased; reached a minimum value at a particular bias; and subsequently increased slightly reaching a second peak and then decreased to near zero as $H_{\mathrm{DC}}$ was further increased. One can also clearly see that the maximum value of $\alpha_{\text {nonlin }}^{\mathrm{ME}}$ at

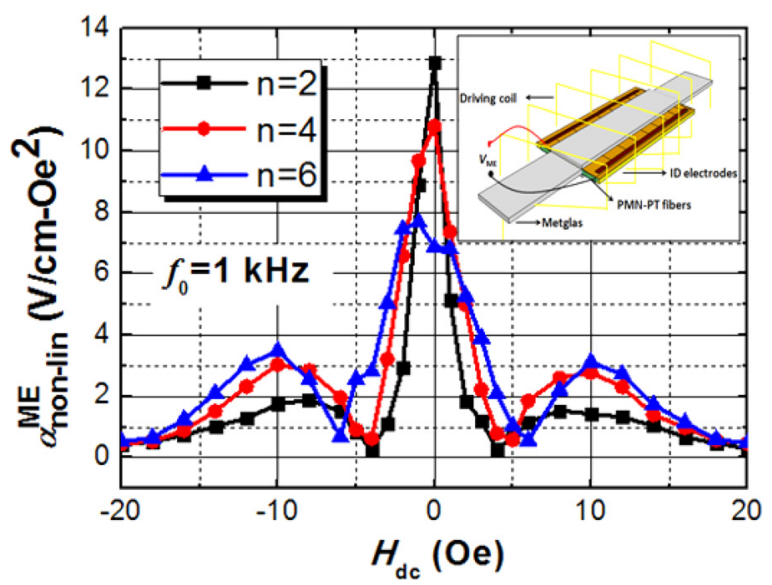

FIG. 1. Non-linear ME coefficient $\alpha_{\text {nonlin }}^{\mathrm{ME}}$ as a function of $H_{\mathrm{DC}}$ for various PMN-PT fiber-Metglas laminate composites under $f_{1}=1 \mathrm{~Hz}$. The numbers show the number (n) of Metglas layers stacked on both sides of the piezo-fibers, where $n$ was varied by successively peeling off layers. The inset shows a schematic diagram of the proposed Metglas/PMN-PT composites with driving coil.
$H_{\mathrm{DC}}=0$ increased with decreasing number of Metglas layers until $\mathrm{n}=2$. Moreover, the $H_{\mathrm{DC}}$ required for the second peak in $\alpha_{\mathrm{nonlin}}^{\mathrm{ME}}$ decreased with decreasing $\mathrm{n}$. The enhancement in $\alpha_{\text {nonlin }}^{\mathrm{ME}}$ for $\mathrm{n}=2$ relative to $\mathrm{n}=6$ is noteworthy, as prior studies of passive ME sensors have been focused on $n=6$ to achieve a maximum linear ME coefficient $\alpha^{\mathrm{ME}} \cdot{ }^{14-16}$ The value of $\alpha_{\text {nonlin }}^{\mathrm{ME}}$ at $H_{\mathrm{dc}}=0$ was determined to be 13 $\mathrm{V} /\left(\mathrm{cm}-\mathrm{Oe}^{2}\right)$ for $\mathrm{n}=2$, which represents an improvement of 6 times over previously reported values. ${ }^{12}$

Figure 2(a) shows the linear ME voltage coefficient $\alpha^{\mathrm{ME}}$ for the same structures for different values of n. $\alpha^{\mathrm{ME}}$ was measured using a lock-in amplifier, as a function of $H_{\mathrm{DC}}$ and in response to $H_{\mathrm{AC}}=1 \mathrm{Oe}$ at $f=1 \mathrm{kHz}$ that was driven by a Helmholtz coil. With increasing $H_{\mathrm{DC}}, \alpha^{\mathrm{ME}}$ increased quickly
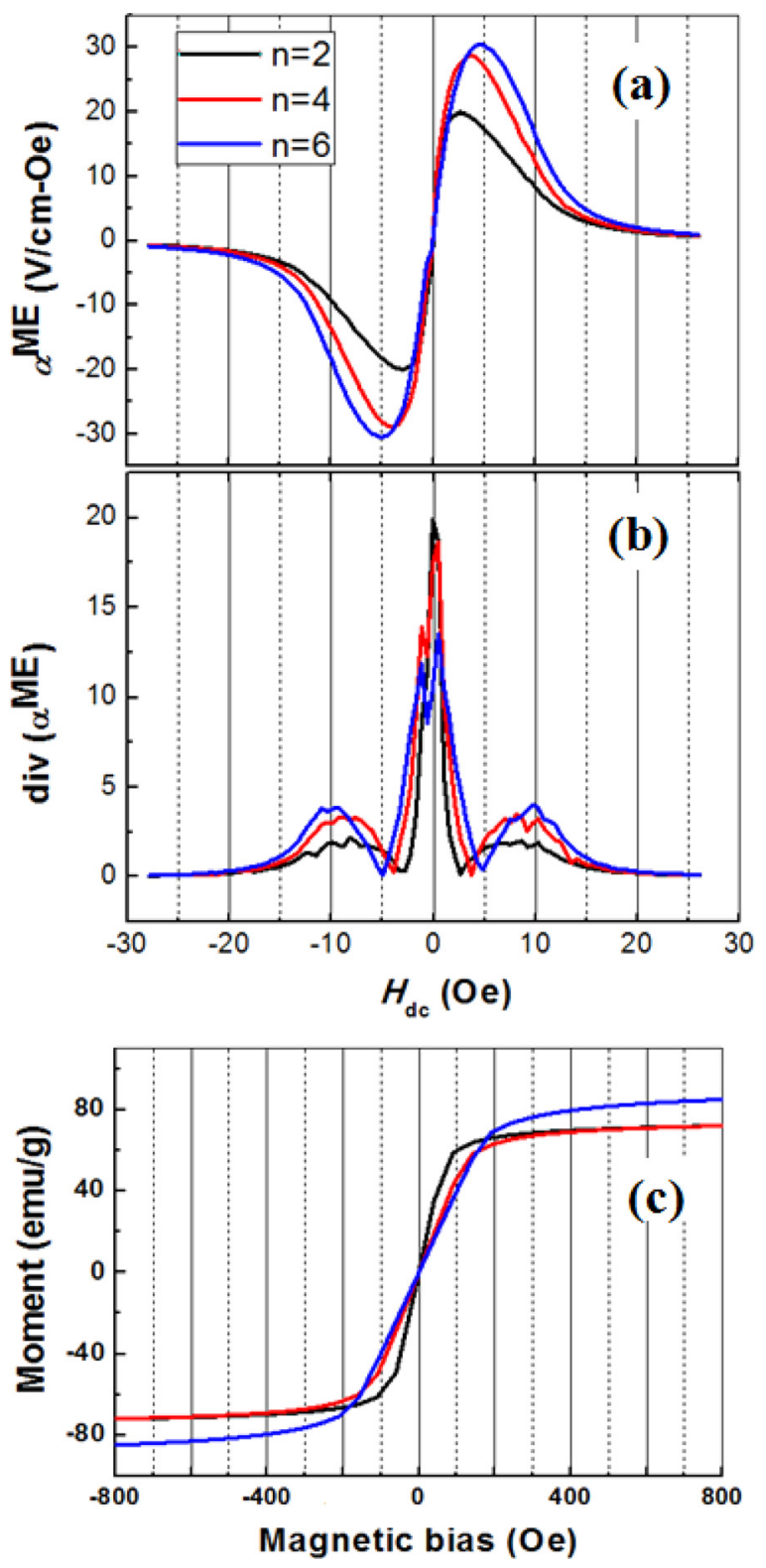

FIG. 2. (a) ME voltage coefficient $\alpha^{\mathrm{ME}}$ as a function of $H_{\mathrm{DC}}$ for various PMN-PT/Metglas laminates with different Metglas layers; (b) derivative values of ME coefficient $\operatorname{div}\left(\alpha^{\mathrm{ME}}\right)$ as a function of $H_{\mathrm{dc}}$; and (c) magnetization (M) of various Metglas layers as a function of magnetic field applied along the longitudinal direction. 
from zero at $H_{\mathrm{DC}}=0$ to a maximum value at a particular $H_{\mathrm{DC}}$ (which is referred as $H_{\text {optim }}$ ) and then declined to zero for $H_{\mathrm{DC}} \geq 20$ Oe. Such a thickness fraction-dependent $\alpha^{\mathrm{ME}}$ for bimorph heterostructures has previously been shown. ${ }^{14,17,18}$ Please note that the $H_{\text {optim }}$, where $\alpha^{\mathrm{ME}}$ had maximum values for various $\mathrm{n}$, was nearly equal to the bias at which $\alpha_{\text {nonlin }}^{\mathrm{ME}}$ was minimum (see Fig. 1). Figure 2(b) shows the derivative values of ME coefficient $\operatorname{div}\left(\alpha^{\mathrm{ME}}\right)$ as a function of $H_{\mathrm{DC}}$. A previous study ${ }^{10}$ has indicated that $\alpha_{\text {nonlin }}^{\mathrm{ME}}$ is dependent on the derivative of the piezomagnetic strain and should be proportional to $\operatorname{div}\left(\alpha^{\mathrm{ME}}\right)$, which is consistent with data of Figs. 1 and 2(b). In these figures, one can clearly see that $\mathrm{n}=2$ has the largest maximum $\operatorname{div}\left(\alpha^{\mathrm{ME}}\right)$ value at $H_{\mathrm{DC}}=0$, that the required $H_{\mathrm{DC}}$ decreased with decreasing $\mathrm{n}$ reaching a minimum value near $H_{\mathrm{DC}}=5 \mathrm{Oe}$, and that a second peak occurred near $H_{\mathrm{DC}} \sim 10 \mathrm{Oe}$.

Figure 2(c) shows the magnetization $(M)$ for various $\mathrm{n}$ as a function of magnetic field applied along the longitudinal direction. These data were obtained by using a vibrating sample magnetometer (VSM). The in-plane $M-H$ behaviors for $\mathrm{n}=2,4,6$ show that the required $H_{\mathrm{DC}}$ increased as $\mathrm{n}$ was increased. Thus, the linear magnetic susceptibility $\left(\chi_{\mathrm{m}}\right)$, defined as the differential of the magnetization with magnetic bias $(\partial M / \partial H)$, is the slope of the $M-H$ curves. In this figure, the slope of the $M-H$ curves can be seen to increase with decreasing $\mathrm{n} . \chi_{\mathrm{m}}$ has a significant effect on the relaxation factor $k$, which is proportional to the piezomagnetic coefficient $d_{33}^{m}$, given as ${ }^{19}$

$$
k=\frac{3 \chi_{m}}{M_{s}} \text { and } k \sim d_{33}^{m},
$$

where $M_{\mathrm{s}}$ is the saturation magnetization. The value of $\chi_{\mathrm{m}}$ increased with decreasing $\mathrm{n}$, resulting in an increase of $k$ at a constant $M_{\mathrm{s}}$ and a subsequently enhanced value of $d_{33}^{m}$. Recently, it has been reported that $\alpha_{\text {nonlin }}^{\mathrm{ME}}$ is highly dependent on the derivative of the piezomagnetic strain coefficient, $\alpha_{\text {nonlin }}^{\mathrm{ME}} \propto \partial d_{33}^{m} / \partial H .^{10,20}$ As a consequence, the value of $\alpha_{\text {nonlin }}^{\mathrm{ME}}$ increased with decreasing $\mathrm{n}$ as evidenced in Figure 1.

The demodulated signal at $f_{1}=1 \mathrm{~Hz}$ was then measured by a SR-785 dynamic signal analyzer, where the modulation signal $\left(f_{0} \pm f_{1}\right)$ was multiplied again by the driving signal $\left(f_{0}\right)$ using a SR-850 lock-in amplifier. Figure 3(a) shows the demodulated signal for a ME structure with $n=2$. These data were measured in response to an increasing $H_{\text {inc }}$ between $2 \mathrm{nT}$ and $90 \mathrm{nT}$ at $f_{1}=1 \mathrm{~Hz}$, where $H_{\mathrm{AC}}$ was fixed at $f_{0}=1 \mathrm{kHz}$. In this figure, it can be seen with increasing $H_{\text {inc }}$ that the demodulated signal increased linearly with a transfer function of $3.7 \mathrm{~V} / \mathrm{Oe}$, as shown in Figure 3(b). Further increase in the drive signal frequency to $f_{0}=10 \mathrm{kHz}$ and $24.2 \mathrm{kHz}$ (i.e., the EMR) resulted in transfer functions of 5.3 V/Oe and 33.4 V/Oe, respectively, shown in Figure 3(b). These data were taken at a constant driving signal of $H_{\mathrm{AC}}=70 \mathrm{nT}$ under $H_{\mathrm{DC}}=0$. A tuning resistor was utilized to cancel the inductance of the driving coil, maintaining a stable magnetic field amplitude that was independent of frequency $f_{0}$. As shown in Fig. 3(c), a significantly increased modulation transfer ability at $f_{0}=24.2 \mathrm{kHz}$ was observed, which can be attributed to the high value of $\alpha_{\text {nonlin }}^{\mathrm{ME}}=100$ $\mathrm{V} /\left(\mathrm{cm}-\mathrm{Oe}^{2}\right)$ at $H_{\mathrm{DC}}=0$ under the drive conditions. Clearly, the frequency transfer ability is dependent on the non-linear ME coefficient.

The voltage spectrum noise density of the demodulated signal was then measured using a SR-785 dynamic signal analyzer in units of $\mathrm{V} / \sqrt{ } \mathrm{Hz}$. These data were obtained in response to a fixed drive signal of $H_{\mathrm{AC}}=70 \mathrm{nT}$ at different frequencies of $f_{0}=1,10$, and $24.2 \mathrm{kHz}$. Upon applying the transfer function, the equivalent magnetic noise spectrum can be converted into units of $\mathrm{T} / \sqrt{ } \mathrm{Hz}$, as follows:
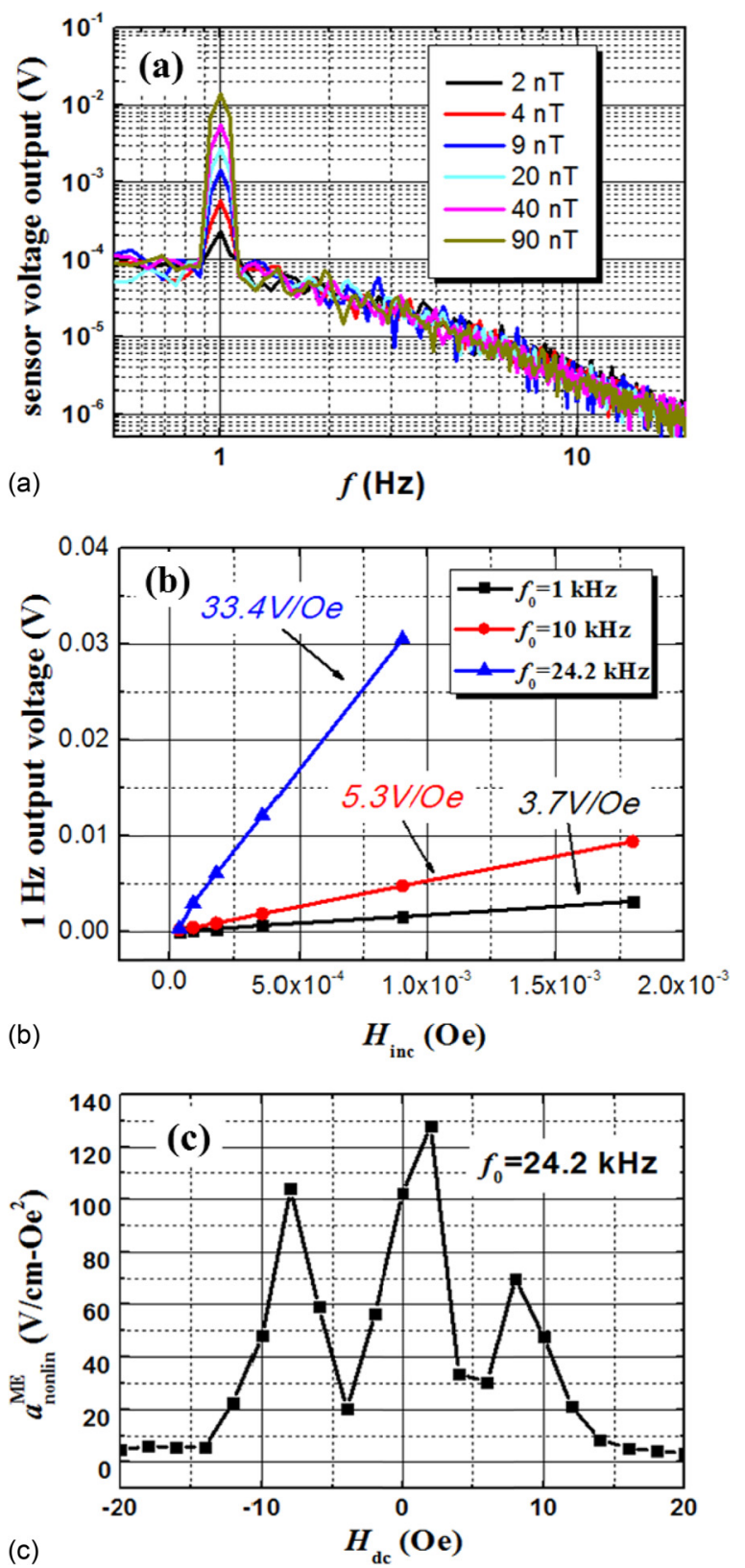

FIG. 3. (a) Signal for a structure with $\mathrm{n}=2$ in response $2 \leq H_{\text {inc }} \leq 90 \mathrm{nT}$ at $f_{1}=1 \mathrm{~Hz}$ under zero DC bias, where $H_{\mathrm{AC}}=70 \mathrm{nT}$ at $f_{0}=1 \mathrm{kHz}$; (b) linear frequency modulation transfer function of $3.7 \mathrm{~V} / \mathrm{Oe}, 5.3 \mathrm{~V} / \mathrm{Oe}$, and $33.4 \mathrm{~V} / \mathrm{Oe}$ for $f_{0}=1,10$, and $24.2 \mathrm{kHz}$ (EMR of the laminates); (c) non-linear ME coefficient $\alpha_{\text {nonlin }}^{\mathrm{ME}}$ as a function of $H_{\mathrm{DC}}$ for Metglas/PMN-PT laminate composites with $\mathrm{n}=2$ for $f_{1}=24.2 \mathrm{~Hz}$. 


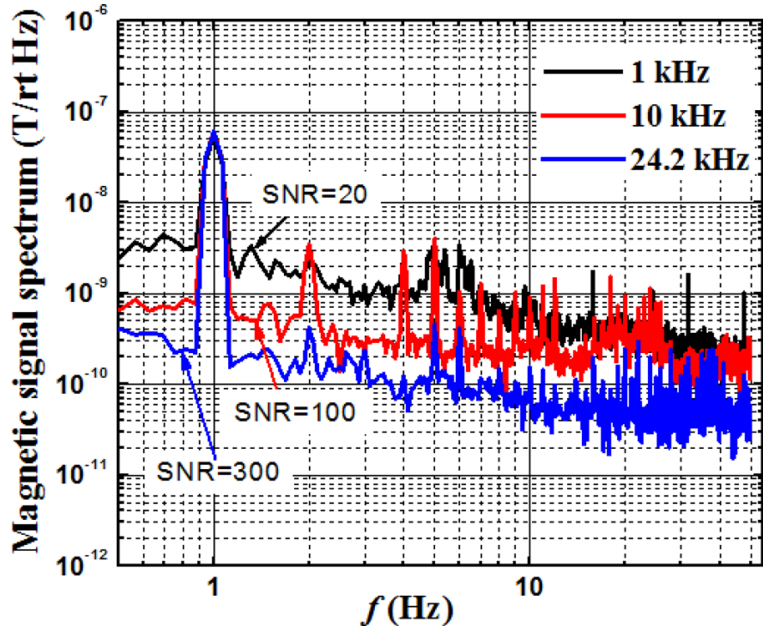

FIG. 4. Equivalent magnetic noise spectrum for a magnetic signal at $1 \mathrm{~Hz}$, after applying modulation transfer functions at various driving frequencies at $f_{0}=1,10$, and $24.2 \mathrm{kHz}$. The noise floor can be seen to be $0.2 \mathrm{nT} /{ }_{ } \mathrm{Hz}$ at $1 \mathrm{~Hz}$ for $f_{0}=24.2 \mathrm{kHz}$. When $f_{0}=24.2 \mathrm{kHz}$, the highest signal to noise ratio was reached with $\mathrm{SNR}=300$, compared to $\mathrm{SNR}=20$ and 100 for $f_{0}=1$ and $10 \mathrm{kHz}$. This offers the potential to allow detection of weaker magnetic fields.

$$
\begin{aligned}
& \text { Equivalent magntic noise }\left(\frac{\mathrm{T}}{\sqrt{\mathrm{Hz}}}\right) \\
& =\frac{\text { voltage noise }\left(\frac{\mathrm{V}}{\sqrt{\mathrm{Hz}}}\right)}{\text { transfer function }\left(\frac{\mathrm{V}}{\mathrm{Oe}}\right)} \times 10^{-4} .
\end{aligned}
$$

Figure 4 shows a representative equivalent magnetic noise spectrum. In this figure, the noise floor can be seen to be about $0.2 \mathrm{nT} / \sqrt{ } \mathrm{Hz}$ at $1 \mathrm{~Hz}$ for $f_{0}=24.2 \mathrm{kHz}$, which was much lower than that of $3 \mathrm{nT} /{ }_{\sqrt{H z}}$ for $f_{0}=1 \mathrm{kHz}$. This decrease in the equivalent magnetic noise floor is a direct consequence of the use of a high driving frequency (at $f_{0}$ ) which can effectively transfer a low frequency target signal $\left(f_{1}\right)$ to higher frequencies $\left(f_{0} \pm f_{1}\right)$, where the noise floor is much lower. It should be noted that these measurements were performed using a constant incident signal of $H_{\text {inc }}=18$ nT at $f_{1}=1 \mathrm{~Hz}$, as can be identified in the signal strengths to be constant as $f_{0}$ was varied in Fig. 4 . The constant signal strength and reduced noise floor together resulted in an increased SNR at $f_{0}=24.2 \mathrm{kHz}$. The SNR reached values of 300 , which were significantly higher than SNR $=20$ and 100 for $f_{0}=1$ and $10 \mathrm{kHz}$. A higher value of SNR offers the potential to allow detection of much weaker magnetic fields. Thus, the enhanced SNR found by optimizing $f_{0}$ to be close to the EMR condition of the ME laminates offers promise for active mode ME sensor applications, which takes advantage of the enhanced values of $\alpha_{\text {nonlin }}^{\mathrm{ME}}$ using the optimized thickness ratio and EMR drive (see Figs. 3(c) and 4). Significantly lower equivalent magnetic noise floors have been obtained in this study, consistent with prior predictions. $^{12}$

\section{CONCLUSION}

In summary, a decrease in the number of Metglas layers has been shown to result in an enhancement in the non-linear ME coefficient at $H_{\mathrm{DC}}=0$ for Metglas/PMN-PT based laminate composites. Using a drive signal at $f_{0}=1 \mathrm{kHz}$, a high value of $\alpha_{\text {nonlin }}^{\mathrm{ME}}=13 \mathrm{~V} /\left(\mathrm{cm}-\mathrm{Oe}^{2}\right)$ was obtained for $\mathrm{n}=2$, which was further increased to $\alpha_{\text {nonlin }}^{\mathrm{ME}}=100 \mathrm{~V} /\left(\mathrm{cm}-\mathrm{Oe}^{2}\right)$ at $f_{0}=\mathrm{EMR}$. These findings demonstrate much higher frequency modulation efficiencies with $\mathrm{SNR}=300$ and lower noise floors.

${ }^{1}$ M. Bibes and A. Barthélémy, Nature Mater. 7, 425-426 (2008).

${ }^{2}$ W. Eerenstein, N. D. Mathur, and J. F. Scott, Nature 442(7104), 759-765 (2006)

${ }^{3}$ H. Greve, E. Woltermann, H.-J. Quenzer, B. Wagner, and E. Quandt, Appl. Phys. Lett. 96(18), 182501 (2010).

${ }^{4}$ S. M. Gillette, A. L. Geiler, D. Gray, D. Viehland, C. Vittoria, and V. G. Harris, IEEE Magn. Lett. 2, 2500104 (2011).

${ }^{5}$ J. Petrie, D. Gray, D. Viehland, G. Sreenivasulu, G. Srinivasan, S. Mandal, and A. S. Edelstein, J. Appl. Phys. 111(7), 07 C714 (2012).

${ }^{6}$ X. Zhuang, M. L. C. Sing, C. Cordier, S. Saez, C. Dolabdjian, L. Shen, J. F. Li, M. Li, and D. Viehland, IEEE Sens. J. 11(10), 2266-2272 (2011).

${ }^{7}$ J. Ma, Z. Li, Y. Lin, and C. W. Nan, J. Magn. Magn. Mater. 323(1), 101-103 (2011).

${ }^{8}$ W. Zhang, G. Yin, J. Cao, J. Bai, and F. Wei, Appl. Phys. Lett. 100(3), 032903 (2012).

${ }^{9}$ J. Gao, Y. Shen, P. Finkel, J. Blottman, J. Li, and D. Viehland, Mater. Lett. 88, 47-50 (2012).

${ }^{10}$ Y. Shen, J. Gao, Y. Wang, P. Finkel, J. Li, and D. Viehland, Appl. Phys. Lett. 102(17), 172904 (2013).

${ }^{11}$ S. Dong, J. Zhai, F. Bai, J.-F. Li, and D. Viehland, Appl. Phys. Lett. 87(6), 062502 (2005).

${ }^{12}$ L. Shen, M. Li, J. Gao, Y. Shen, J. F. Li, D. Viehland, X. Zhuang, M. L. C. Sing, C. Cordier, S. Saez, and C. Dolabdjian, J. Appl. Phys. 110(11), 114510 (2011).

${ }^{13}$ S. Dong, J. Zhai, J. Li, and D. Viehland, Appl. Phys. Lett. 89(25), 252904 (2006).

${ }^{14}$ J. Das, J. Gao, Z. Xing, J. F. Li, and D. Viehland, Appl. Phys. Lett. 95(9), 092501 (2009).

${ }^{15}$ J. Gao, Z. Wang, Y. Shen, M. Li, Y. Wang, P. Finkel, J. Li, and D. Viehland, Mater. Lett. 82, 178-180 (2012).

${ }^{16}$ Y. Wang, D. Gray, D. Berry, J. Gao, M. Li, J. Li, and D. Viehland, Adv. Mater. 23(35), 4111-4114 (2011).

${ }^{17}$ Y. Wang, J. Gao, M. Li, D. Hasanyan, Y. Shen, J. Li, D. Viehland, and H. Luo, Appl. Phys. Lett. 101(2), 022903 (2012).

${ }^{18}$ F. Fang, C. Zhao, and W. Yang, Sci. China: Phys., Mech. Astron. 54(4), 581-585 (2011).

${ }^{19}$ X. J. Zheng and X. E. Liu, J. Appl. Phys. 97(5), 053901 (2005).

${ }^{20}$ D. A. Burdin, D. V. Chashin, N. A. Ekonomov, Y. K. Fetisov, L. Y. Fetisov, G. Sreenivasulu, and G. Srinivasan, J. Appl. Phys. 113(3), 033902 (2013). 